\title{
A New Prior Shape Model for Level Set Segmentation
}

\author{
Poay Hoon Lim ${ }^{1}$, Ulas Bagci ${ }^{2}$, and $\mathrm{Li} \mathrm{Bai}^{1}$ \\ 1 School of Computer Science, University of Nottingham, Nottingham, NG8 1BB \\ phl@cs.nott.ac.uk, bai@cs.nott.ac.uk \\ 2 Radiology and Imaging Science Department, NIH, MD 20892 \\ ulas.bagci@nih.gov
}

\begin{abstract}
Level set methods are effective for image segmentation problems. However, the methods suffer from limitations such as slow convergence and leaking problems. As such, over the past two decades, the original level set method has been evolved in many directions, including integration of prior shape models into the segmentation framework. In this paper, we introduce a new prior shape model for level set segmentation. With a shape model represented implicitly by a signed distance function, we incorporate a local shape parameter to the shape model. This parameter helps to regulate the model fitting process. Based on this local parameter of the shape model, we define a shape energy to drive the level set evolution for image segmentation. The shape energy is coupled with a Gaussian kernel, which acts as a weight distribution on the shape model. This Gaussian effect not only allows evolution of level set to deform around the shape model, but also provides a smoothing effect along the edges. Our approach presents a new dimension to extract local shape parameter directly from the shape model, which is different from previous work that focused on an indirect manner of feature extractions. Experimental results on synthetic, optical and MR images demonstrate the feasibility of this new shape model and shape energy.
\end{abstract}

Keywords: image segmentation, level set method, prior shape model, shape energy.

\section{Introduction}

Image segmentation is fundamental to image understanding. Although region and boundary-based segmentation methods have been implemented successfully in many physical applications, these classical methods still not fully utilize the image information to achieve their purposes, for example, in clinical applications. Since its introduction by Osher and Sethian [10, the level set methods have been widely used for image segmentation. For highly challenging segmentation tasks such as tracking moving objects, segmenting occluded scenes and objects of interest from medical images, level set methods have achieved promising results when coupled with prior knowledge or prior shape models [6], [7, 12, 14, 16]. When information such as gradient is missing from images, the prior shape 
helps level set evolve toward the desired region of interest. The segmentation is determined by a dissimilarity measure between the evolving level set function and the prior shape. Different shape models and shape representations have been proposed to couple with the level set methods over the past 10 years. For example, Leventon et al. 9] suggested the representation of a set of training shapes by the principal component of their signed distance function; Tsai [16] proposed to carry out optimization directly within the subspace of the first few eigenmodes. Other examples of shape models for level set can be found in Rousson and Paragios [12, 13. However, these models suffer from shortcomings such as the predefined statistical data distribution might be invalid because it differs from the actual data distribution [5], or modeling non-linear variability of the data with linear methods may not be admissible etc. A more comprehensive review on statistical approaches on integrating shape for level set segmentation is discussed by Cremer et al [8].

In this paper, we introduce a new shape model and an associated shape energy for level set segmentation. Our inspirations come from the work presented by Rousson and Paragios 14. While Rousson introduces a confidence map to identify the reliability of shape fitting process during level set evolution, we focus on extracting local statistical properties from the shape model to enhance the level set evolution. Our segmentation framework consists of two new features: a local shape variance and a kernel weighted functional.

\section{The Segmentation Framework}

\subsection{Level Set Method}

The level set technique, also known as the implicit deformable model, is by embedding the interface in a higher dimensional scalar function. The interface is represented implicitly as a level set (usually the zero-th level set) of the introduced scalar function. The rest of the scalar function is defined as the signed distance function from the interface, i.e., the level set.

Suppose that the level set $\phi(x)$ is evolving in time, i.e., $\phi(x(t), t)=0$. Taking derivative of the last equation with respect to $t$ yields

$$
\frac{\partial \phi}{\partial t}+\nabla \phi(x(t), t) \cdot x^{\prime}(t)=0
$$

As the rate of change of $x(t)$ is in the normal direction of the surface, $\boldsymbol{n}=\frac{\nabla \phi}{|\nabla \phi|}$, one can rewrite the equation as

$$
\frac{\partial \phi}{\partial t}+F|\nabla \phi|=0
$$

where $F=x^{\prime}(t) \cdot \frac{\nabla \phi}{|\nabla \phi|}$ represents the speed function. In recent development of level set function, the "variational level set method" is introduced: An energy $E(\phi)$ is defined in relation to the the speed function. The minimization of 
such energy which generates the Euler-Lagrange equation, provides the evolution equation through the calculus of variation:

$$
\frac{\partial \phi}{\partial t}=-\frac{\partial E(\phi)}{\partial \phi} .
$$

Our purpose is to integrate shape energy into this evolution equation, i.e., to define a speed function for level set evolution with the introduction of local parameter from a shape model.

\subsection{Prior Shape Model}

Inspired by Rousson and Paragios [14], our prior shape model is constructed from a set of training samples represented implicitly by signed distance functions $\left\{\phi_{1}, \phi_{2}, \ldots, \phi_{N}\right\}$. An initial shape model is obtained by taking the average from these signed distance functions. A re-initialization algorithm [15] is then applied to this initial shape model to approximate a signed distance function, $\phi_{m}$, which becomes the shape model. In our case, $\phi_{m}$ forms a global parameter of the shape model. In order to better represent the local information of the shape model, we use a locally enhance, neighboring dependent variance to capture the local shape features:

$$
\sigma_{l}^{2}=\sum_{x \in\{\phi=0\}} \sum_{x_{i} \in U_{x}} \frac{\left(\phi_{m}\left(x_{i}\right)-\phi_{m}(x)\right)^{2}}{\left|U_{x}\right|}
$$

where $U_{x} \subset \phi_{m}$ is a local window surrounding $x$. This local shape parameter not only maintains the smoothness of the shape model, but also incorporates the local features of the shape. The local variance taken around a pixel-wise neighborhood in the shape model provides an insight into the localized properties on the signed distance function: larger variance indicates a larger average distance difference, i.e., a steeper change in the level set function. This has a better physical representation in comparison to the variance obtained for voxel-wise approach in the training samples.

\subsection{The Shape Energy}

Global variance and variance for shape model were used in various work in the past. However, these variances might not reflect the desired shape model information accurately due to variations such as ill alignment of the training samples, wide range of scaling etc. Under certain extreme circumstances, such variations might jeopardize the accuracy of level set evolution. Bear in mind of these limitations, we propose a regularized factor to accompany the energy that incorporates local statistical features. This regularized term is extracted from the prior shape model by taking into account the local average of signed distance function. To achieve our purpose, we use a pixel-wise local statistical variance from the shape model, $\sigma_{l}^{2}$. This is in line with the localization of the shape model 
whereby information is integrating from local neighborhood. The localization along the level set shall enhance the control of the edge based stopping functional.

To formulate the shape energy, we adopt the symmetric dissimilarity measure between two shapes proposed by Chan and Zhu [3], Riklin-Raviv [1], Charpiat [4:

$$
D\left(\phi, \phi_{m}\right)=\int_{\Omega} \frac{1}{2}\left(H(\phi)-H\left(\phi_{m}\right)\right)^{2} d x
$$

where $H(\phi)$ is the Heaviside function. This is then incorporated with an external energy that drive the zero level set towards the object boundary [1]:

$$
E_{1}(\phi)=\lambda \int_{\Omega} g \delta(\phi)|\nabla \phi| d x
$$

where $g$ is the edge indicator function $g=\frac{1}{1+\left|\nabla G_{\sigma} * I\right|^{2}}$, and $G_{\sigma}$ is the Gaussian kernel with standard deviation $\sigma$.

The total energy is thus

$$
\begin{aligned}
E(\phi) & =D\left(\phi, \phi_{m}\right)+E_{1}(\phi) \\
& =\int_{\Omega} \frac{1}{2}\left(H(\phi)-H\left(\phi_{m}\right)\right)^{2} d x+\lambda \int_{\Omega} g \delta(\phi)|\nabla \phi| d x .
\end{aligned}
$$

By taking the Gâteaux derivative of $E(\phi)$, followed by the gradient decent flow that minimizes the functional

$$
\frac{\partial \phi}{\partial t}=-\frac{\partial E}{\partial \phi}
$$

we obtain the standard evolution equation

$$
\frac{\partial \phi}{\partial t}=-\left(H(\phi)-H\left(\phi_{m}\right)\right) \delta(\phi)-\lambda \delta(\phi) d i v\left(g \frac{\nabla \phi}{|\nabla \phi|}\right) .
$$

Now, to incorporate the regularizing effect from shape model's local parameter into the level set evolution, we consider a variation of the above evolution equation by multiplying it with a weighted constraint:

$$
w(\phi)=\frac{1}{2 \sigma_{l}^{2}} e^{-\left(H(\phi)-H\left(\phi_{m}\right)\right)^{2} / 2 \sigma_{l}^{2}}
$$

where $\sigma_{l}^{2}$ is the local variance along the level set. Note that $w(\phi)$ acts as a "weighing" function giving the evolution term $\left(H(\phi)-H\left(\phi_{m}\right)\right) \delta(\phi)$ higher influence when the difference between the level set and the shape model is larger. Hence, our level set evolution equation is

$$
\frac{\partial \phi}{\partial t}=-\frac{1}{2 \sigma_{l}^{2}} e^{-\left(H(\phi)-H\left(\phi_{m}\right)\right)^{2} / 2 \sigma_{l}^{2}}\left(\left(H(\phi)-H\left(\phi_{m}\right)\right) \delta(\phi)+\lambda \delta(\phi) d i v\left(g \frac{\nabla \phi}{|\nabla \phi|}\right)\right) .
$$




\section{Experimental Results}

We implement the proposed framework on synthetic noisy images and optical images with partial blurring effect as well as MR images of spine. For synthetic images, we created five synthetic images of various sizes and aspect ratios as training samples while for optical and MR images, we use four and six training samples respectively. The classical edge based [1] and region based methods [2] are used to compare and to highlight the effectiveness of our approach.

In Fig. 1, we show the shape model and local shape variance of the sample images. The zero level set or shape contour are outlined from the signed distance function images to show the shape model used in our experiments. The contour of training samples are plotted on the extracted local shape parameter images. Images of voxel-wise variance on the shape models are illustrated here for the purpose of comparison with our proposed local variance information. Unlike the
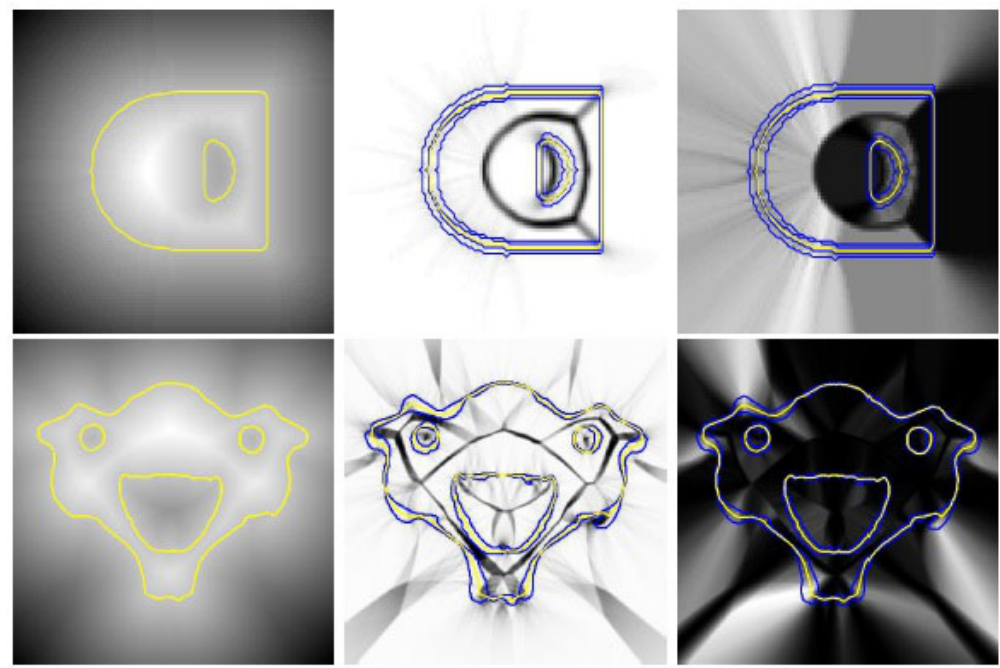

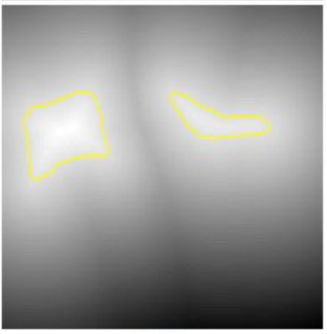

(a)

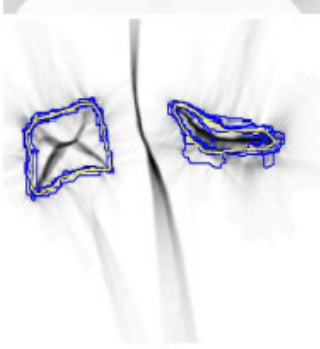

(b)

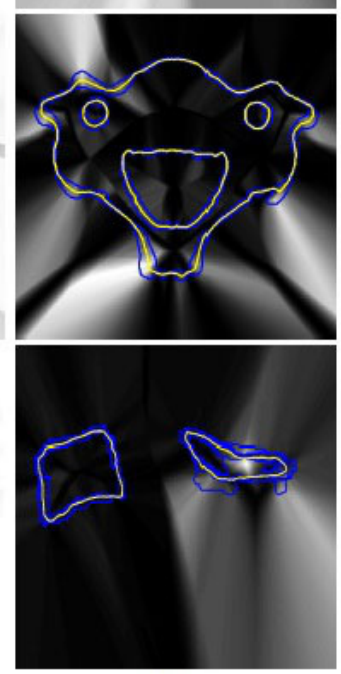

(c)

Fig. 1. The shape model is outlined in red while the training samples are outlined in blue. (a) The shape model and (b) variance local used in our proposed prior shape model for synthetic image (top), optical image (middle) and MR image of spine (bottom) respectively. (c) The voxel-wise variance are shown as comparison to our proposed local shape parameter. 
voxel-wise variance, this local shape parameter highlights the local variations of shape model without creating unwanted excessive variation effects outside the shape model neighborhoods, which happens to the voxel-wise variance. The absence of these excessive side effect helps to ensure the stability of this local shape parameter when applying to level set evolutions.

Fig. 2 demonstrates results obtained from the edge based, region based and shape based approaches respectively. A well known fact on the edge based approach is its sensitivity to initial contour placement, this can be observed from the MR image sample, where part of the image contour is badly located when the initial contour is placed across the edge of region of interest. In addition, even when the initial contour is placed inside the region of interest, the segmentation result is still not as accurate comparing to the results from our proposed shape based approach. Obviously the segmentation for synthetic and optical images by edge based method are not effective because the method cannot handle blurring edges. Although the region based is not sensitive to initial contour placement, the inhomogeneity of image intensities has caused the mal performance of the method on MR image and it is not accurate on blurring edges, as
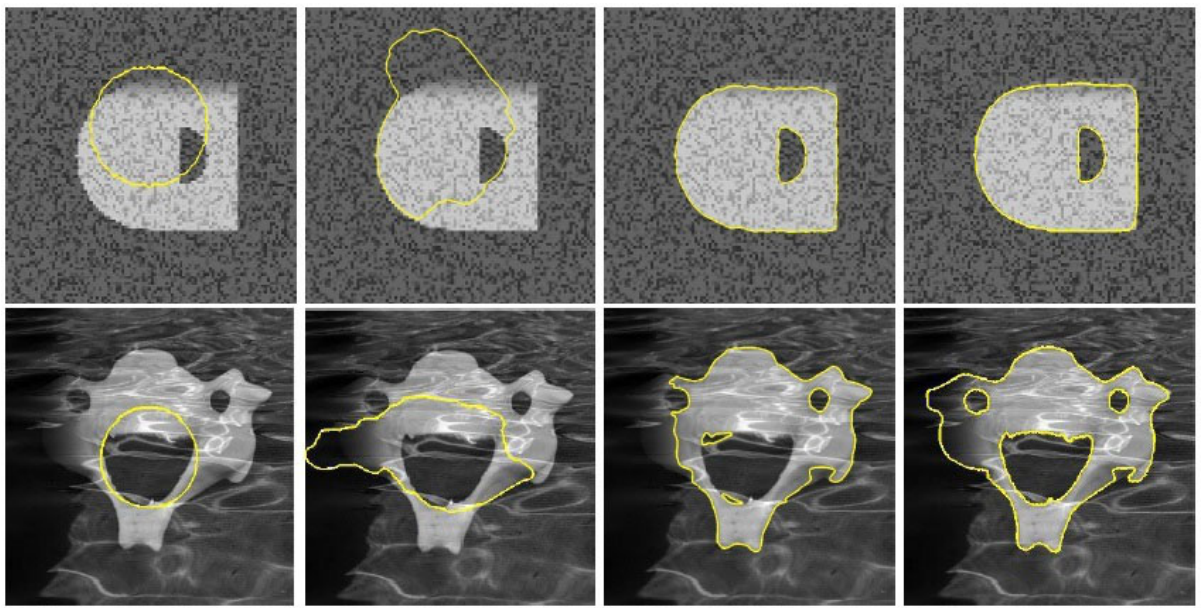

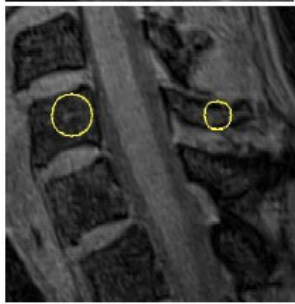

(a)

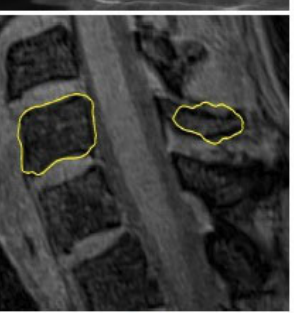

(b)

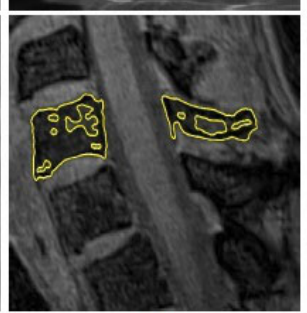

(c)

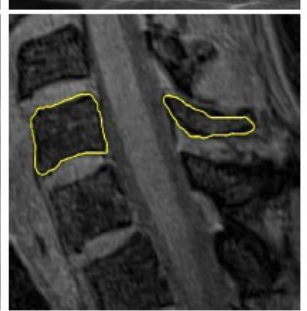

(d)

Fig. 2. (a) The initial contour placement used by all the methods for segmentation. Results obtained by (b) edge based method, (c) region based method and (d) our proposed shape prior based method for synthetic image (top), optical image (middle) and MR image of spine (bottom). 
seen on synthetic and optical images. Whereas results from our shape based approach clearly highlight the region of interest in all sample images and achieved an average accuracy of $93 \%$ when tested on fifteen sample images.

\section{Conclusion}

We have proposed a new image segmentation framework encompassing area dissimilarity, shape model and local variance. Our shape model guides the level set evolution through the introduction of a local shape parameter, together with a new shape energy. The shape parameter provides localized information that acts as a moderator for the shape model and in the level set evolution process. Although our work is inspired by by Rousson and Paragios [14, we take a different path in our approach. Unlike their work whereby emphasis is on capturing the shape model reliability and alignment during level set evolution, we look closer into the local property of shape model and to incorporate it into the segmentation process. In particular, we focus on integrating the local variance of shape model into the level set evolution for regularizing purposes. The proposed segmentation framework has been tested on synthetic images with added noise and blurring effect, as well as on MR images of spine. Experimental results on these images are promising. We merely use one local feature for this work, i.e., the local variance of shape model. Future work is to explore and encompass more information from the shape model, for example, the local geometrical features and to couple it into the level set segmentation framework. Although similar work has been carried out in the past, somehow the information is extracted in an indirect manner, i.e., through the principal component analysis of shape model, and the extracted information are mostly in global sense. With a more direct approach in acquiring information from shape model, we anticipate to obtain better shape parameters for level set segmentation.

Acknowledgments. The authors would like to thank Professor Jayaram K. Udupa of Department of Radiology, University of Pennsylvania for the medical data and advice.

\section{References}

1. Caselles, V., Kimmel, R., Sapiro, G.: Geodesic active contours. International Journal of Computer Vision 22(1), 61-79 (1997)

2. Chan, T.F., Vese, L.A.: Active contours without edges. IEEE Transactionson Image Processing 10(2), 266-277 (2001)

3. Chan, T., Zhu, W.: Level set based shape prior segmentation. Technical Report 03-66, Computational Applied Mathematics, UCLA, Los Angeles, USA (2003)

4. Charpiat, G., Faugreas, O., Keriven, R.: Approximations of shape metrics and application to shape warping and empirical shape statistics. Journal of Foundations of Computational Mathematics 5(1), 1-58 (2005)

5. Cremers, D., Kohlberger, T., Schnorr, C.: Shape Statistics in Kernel Space for Variational Image Segmentation. Pattern Recognition 36(9), 1929-1943 (2003) 
6. Cremers, D., Osher, S.J., Soatto, S.: Kernel Density Estimation and Intrinsic Alignment for Shape Priors in Level Set Segmentation. International Journal of Computer Vision 69(3), 335-351 (2006)

7. Cremers, D.: Dynamical statistical shape priors for level set based tracking. IEEE Transaction on Pattern Analysis and Machine Intelligence 28(8), 1262-1273 (2006)

8. Cremers, D., Rousson, M., Deriche, R.: A Review of Statistical Approaches to Level Set Segmentation: Integrating Color, Texture, Motion and Shape. International Journal of Computer Vision 72(2), 195-215 (2007)

9. Leventon, M., Grimson, E., Faugeras, O.: Level set based segmentation with intensity and curvature priors. IEEE Mathematical Methods in Biomedical Image Analysis, 4-11 (2000)

10. Osher, S., Sethian, J.A.: Fronts propagating with curvature-dependent speed: Algorithms based on Hamilton-Jacobi formulations. Journal of Computational Physics 79(1), 12-49 (1988)

11. Riklin-Raviv, T., Kiryati, N., Sochen, N.: Unlevel-sets: Geometry and prior-based segmentation. In: Pajdla, T., Matas, J(G.) (eds.) ECCV 2004, Part IV. LNCS, vol. 3024, pp. 50-61. Springer, Heidelberg (2004)

12. Rousson, M., Paragios, N.: Shape priors for level set representations. In: Heyden, A., Sparr, G., Nielsen, M., Johansen, P. (eds.) ECCV 2002, Part II. LNCS, vol. 2351, pp. 78-92. Springer, Heidelberg (2002)

13. Rousson, M., Paragios, N., Deriche, R.: Implicit active shape models for $3 \mathrm{~d}$ segmentation in MRI imaging. In: Barillot, C., Haynor, D.R., Hellier, P. (eds.) MICCAI 2004, Part I. LNCS, vol. 3216, pp. 209-216. Springer, Heidelberg (2004)

14. Rousson, M., Paragios, N.: Prior Knowledge, Level Set Representations \& Visual Grouping. Int. J. Comput. Vis. 76, 231-243 (2008)

15. Sussman, M., Smereka, P., Osher, S.: A level Set Approach for Computing Solutions to Incompresible Two-Phase Flow. Journal of Computational Physics 114, 146-159 (2002)

16. Tsai, A., Yezzi Jr., A., Wells, W., Tempany, C., Tucker, D., Fan, A., Grimson, W., Willsky, A.: A Shape-Based Approach to The Segmentation of Medical Imagery Using Level Sets. IEEE Trans. on Medical Imaging 22(2), 137-154 (2003) 